The Genetics of Coeliac Disease

Edited by R B McConnell. (Pp xxxi +301 ; figures + tables. £24.95.) Lancaster, Boston, The Hague: MTP Press. 1981.

Gluten sensitive enteropathy (GSE) has great interest for clinical geneticists because, unlike diabetes and most other HLA associated putative autoimmune diseases, an avoidable environmental agent is known. Strict adherance to a gluten free diet (GFD), especially in children, completely reverses the sub-total villous atrophy of jejunal mucosa and restores normal health. The association of GSE with HLA is stronger than between HLA and insulin dependent diabetes, and sibs with GSE generally share both or at least one of the parental HLA haplotypes. Linkage between genes for HLA and resistance to GSE is thus highly probable, but there are intriguing suggestions of two locus causation involving interaction between MHC and non-HLA antigens on B-lymphocytes. Thus, a package of oligogenic variance in response to gluten has displaced concepts involving multifactorial determination, an increasingly familiar theme in the genetics of common diseases. Empirically, family studies using jejunal biopsy as an indicator of GSE show that $10 \%$ of first degree relatives have the disease and rather more have ambiguous lesser anomalies of jejunal mucosa. Only a proportion of those with biopsy evidence of GSE actually seem to suffer from their blunted villi. Why some people who are genetically susceptible and respond pathologically to gluten should be so fortunate is an unresolved (and possibly multifactorial!) phenomenon. There is other evidence of heterogeneity. For example, long term complications include lymphoma with a very bad prognosis, while some patients become refractory to GFD. Epidemiologically, coeliac disease is also fascinating and there is a geographical parallel between the availability of gluten containing cereals, coeliac disease, and the HLA antigens B8, DR3, and DR7.

These topics are well covered in the proceedings of the International Symposium on the Genetics of Coeliac Disease held in the Liverpool Medical Institution on 28 and 29 November 1979. Organised by Richard McConnell, the doyen of gastrointestinal genetics, many of the most active workers in this field were attracted to Liverpool. The Liverpool group itself contributed several chapters to the proceedings. The arcane topics of HLA and MHC linked complement markers are discussed with elegant simplicity by Woodrow. Ellis reviewed previous family studies and in collaboration with others reported on the Liverpool Coeliac Family Study which has contributed much to the genetics of this disease. The epidemiology of coeliac disease and its remarkable variation in frequency in different parts of the world leads Edwards, with many a genetic bon mot, to conclude that the elimination of coeliac disease might follow genetic counselling offered to the breeders of cereals. Several chapters devoted to genetics address the popular problem of the control of gene penetrance in common diseases, while Warren Strober neatly encapsulates current concepts of pathogenesis involving HLA and interaction with a putative second susceptibility locus for GSE.

Sir Cyril Clarke, in his introductory lecture on the wider horizon, asks whether patients benefit from our knowledge of HLA, an important question considering the resources that have been devoted to this popular genetic system. Leaving aside the major, albeit patchy, achievements of tissue typing for organ transplantation, it is difficult yet to discern a direct benefit. However, Albert and Booth remind us of the need for academic research, not solely concerned with cui bono. In his 1981 Nobel Prize Lecture in another place, Benacerraf said in the context of MHC: "The identification of the genes that determine biological phenomena and the study of the control they exert on these phenomena has proved to be the most successful approach to a detailed understanding of the mechanism of biological processes". Surely the genes that determine the pathological response to gluten are worthy of study, especially since control of coeliac disease is achieved by removal of dietary gluten. The essence of ecogenetics is precisely the identification of genetic susceptibility to exogenous agents and prevention by selective manipulation of the environment. Who knows what generally applicable lessons we may learn by the study of the uniquely polymorphic major histocompatibility complex.

RODNEY HARRIS

\section{Principles of Gene Manipulation. An Introduction to Genetic Engineering}

2nd ed. Edited by R W Old and S B Primrose. (Pp x + 214; figures + tables. £6.50.) Oxford: Blackwell Scientific Publications. 1981.

Two years ago, the publication of Principles of Gene Manipulation provided students with a straightforward introduction to genetic engineering. The technological advances since then are important and the updated edition of this book is therefore useful. The basic principles of gene manipulation are clearly explained, as in the first edition, and there 
are extra sections included where the field has advanced significantly.

Cloning in the single-stranded vector M13 and its application to DNA sequencing is described. It is a pity that the Maxam-Gilbert technique of DNA sequencing is not discussed anywhere in the text. The sequence analysis of eukaryotic gene fragments, particularly by the latter method, has played an important role in our understanding of gene structure and function. A paragraph comparing the applications and limitations of both these sequencing methods would have been valuable.

A new chapter has been included encompassing cloning principles in systems other than $E$ coli, such as Bacillus subtilis and yeast. The chapter on cloning in plant cells has also been extended in a constructive way. This account provides an excellent introduction to an application of gene manipulation which will be very important in the next few years.

The major weakness of the text is in its depiction of the impact of this technology on molecular biology. An attempt is made to summarise the applications in chapter 11 , but it falls short of describing major advances made in human genetics and in the understanding of gene organisation.

In summary, Principles of Gene Manipulation provides an informative introduction to genetic engineering, but should be recommended to students in conjunction with important references describing the applicaition of this technology.

K E DAvies

\section{Clinical Genodermatology}

Volumes 1 and 2. By Thomas Butterworth and Roger L Ladda. (Vol 1: Pp xxi + 374; figures. $£ 35 \cdot 50$. Vol 2: Pp xxi +355 ; figures. £34.50.) New York: Praeger. 1981.

Dermatologists have long awaited a definitive work on genetic diseases of the skin, and it was therefore with some excitement that I first opened this two volume candidate for the title. After that, I met with a series of disappointments. The first was simply the appearance of the book, both externally and internally, which I found tired and old-fashioned. Superficially, it might have been published in 1881 .

The book focuses its attention on single gene disorders with prominent cutaneous manifestations and selection of conditions has been comprehensive. Groupings of disorders are intelligent and the clinical descriptions are, on the whole, excellent. Early manifestations, often ignored, are given appropriate attention. The text is generously illustrated with black and white photographs, but these are too often unhelpful, either because they show atypical features or because they are of low quality. Nomenclature is uncritically handled and will I fear confuse many readers. I found this a real weakness in the book. A number of antique terms are retained, such as adenoma sebaceum for the facial angiofibromata of tuberous sclerosis, which are neither adenomatous nor have anything to do with sebaceous glands. Elsewhere there are more serious mistakes, including the listing of 'synonyms' which are in fact entirely separate conditions. Lamellar ichthyosis of the newborn and harlequin fetus are not the same entity at all. The latter is a single gene disorder incompatible with life, the former simply a phenotype which may occur as the initial manifestation of any one of a number of genetic disorders and which is only rarely a cause of death. The biochemical basis of the diseases discussed is, in my view, given too little attention. It would have been helpful to give definite answers as to whether or not prenatal diagnosis is possible in the case of each disorder. Discussion of the genetic heterogeneity of such disorders as the Ehlers-Danlos syndrome and xeroderma pigmentosum is considered to be beyond the book's scope. Surely it shouldn't be?

Each condition has a sub-heading 'Variations'. This contains, as one would expect, discussions on the range of expression seen in each disease, but in many places it appears to confuse this with variations due to distinct genes. It is generally accepted that there are two distinct forms of epidermolysis bullosa simplex, the so-called generalised form and the Cockayne type, which is localised to the hands and feet. The authors describe the localised form as a variant of the generalised form, without making it clear whether they believe that the same gene is responsible or not. Worse still, they include the so-called acquired type of epidermolysis bullosa as a 'variant' of autosomal recessive dystrophic epidermolysis bullosa, though there is no evidence that the former has any genetic basis whatsoever. Such confusion is unjustifiable anywhere, but it is particularly sad that it should mar a book on a genetic topic.

Each volume has its pages independently numbered and its own separate index. This makes it extremely uncomfortable to use the book for reference purposes. Surely the correct system for a work of this type is to continue page numbering from the first into the second volume, and provide each volume with a copy of a single comprehensive index?

The book is for all these reasons not the one we have been waiting for, but it is nevertheless the best available on the subject and will be bought for this reason.

D J Atherton 